\title{
Diagnóstico ambiental do meio físico: ausência de análises geoquímicas nos relatórios de controle ambiental para obtenção de licença prévia para perfuração de poços de petróleo onshore do Rio Grande do Norte
}

\section{Anna Paula Lima Costa ${ }^{1 *}$ \\ Rosiney Araújo Martins ${ }^{1}$}

1'Instituto Federal de Educação, Ciência e Tecnologia do Rio Grande do Norte (IFRN) Natal (RN), Brasil.

*Autor correspondente: anna.costa@ifrn. edu.br

\begin{abstract}
Resumo
Este estudo tem o objetivo de verificar como a ausência de análises e dados geoquímicos como parte do diagnóstico ambiental do meio físico influencia o processo de licenciamento ambiental aplicado à perfuração de poços de petróleo. A pesquisa é de caráter bibliográfico e documental, resultando em um roteiro dos principais estudos geoquímicos que devem ser realizados nas atividades petrolíferas, bem como dos principais produtos consequentes destes dados geoquímicos. Concluímos que os relatórios de controle ambiental analisados para o processo de obtenção de licença prévia para perfuração de poços de petróleo onshore do Rio Grande do Norte não apresentam por completo as análises e/ou dados geoquímicos necessários para caracterizar o meio físico das atividades petrolíferas, prejudicando, assim, as etapas seguintes de monitoramento e elaboração das medidas mitigadoras.

Palavras-chave: diagnóstico ambiental, meio físico, análises geoquímicas, RCAs, petróleo onshore.
\end{abstract}

\section{Abstract}

This study aims to verify how the absence of analyses and geochemical data as part of the environmental diagnosis of the physical environment influences the environmental licensing process applied to the drilling of oil wells. The research is bibliographic and documentary, resulting in the availability of an itinerary of the main geochemical studies that should be conducted in oil activities, as well as the main products that come from these geochemical data. We have concluded that the reports of environmental control analyzed for the process of obtaining a license prior to the drilling of onshore oil wells in Rio Grande do Norte did not fully present the analyzes and/or geochemical data needed to characterize the physical environment of the oil activities, thus harming the next steps of monitoring and preparation of mitigation measures.

Keywords: environmental diagnosis, physical environment, geochemical analyzes, RCAs, onshore oil. 


\section{INTRODUÇÃO}

O Rio Grande do Norte apresenta um potencial petrolífero favorável que decorre da existência de duas bacias sedimentares de extensão e espessura expressivas, nas quais se destacam as áreas da Bacia Potiguar e do norte da Bacia Pernambuco-Paraíba. Em observância à lei vigente que regula a concessão de áreas visando à pesquisa e à produção de petróleo e gás, a Agência Nacional do Petróleo, Gás Natural e Biocombustíveis (ANP) fiscalizou em 2010 no Estado do Rio Grande do Norte 57 concessões exploratórias e 82 concessões de produção. Dessas, 11 estão em fase de desenvolvimento e 71 em produção (Pfaltzgraff \& Torres 2010).

Diante do potencial petrolífero do Rio Grande do Norte, procuramos destacar a relevância do processo de licenciamento ambiental dessas áreas, ressaltando a importância da qualidade da elaboração dos relatórios de controle ambiental (RCAs). Os três RCAs pesquisados, cedidos pelas empresas Projetos Geológicos Ltda. (PROGEL 2008a, 2008b) e Empresa de Consultoria e Planejamento Ambiental Ltda. (ECOPLAN 2008), não apresentam análises e/ou dados geoquímicos, prejudicando todo o processo de avaliação de impactos ambientais.

O Instituto de Desenvolvimento Econômico e Meio Ambiente (IDEMA), órgão responsável por tal licenciamento, disponibiliza o termo de referência (TR) para elaboração do

\section{ESTUDOS DE IMPACTOS AMBIENTAIS}

Toda e qualquer forma de exploração e uso dos recursos naturais e dos produtos de sua transformação, por mais cautelosa que seja, é potencialmente causadora de poluição ambiental. Como a sobrevivência humana depende da exploração dos recursos naturais da Terra, torna-se necessário conhecer e reconhecer os efeitos da exploração dos recursos naturais no ambiente, não para impedir a poluição, mas para tomar consciência das formas de exploração que conduzam à minimização dos impactos (Matos 2010). Desta forma, para minimizar esses impactos negativos no meio ambiente, é importante a realização do diagnóstico ambiental com a finalidade de melhor avaliar e prevenir as consequências das atividades no ambiente.

São definidos pelo Art. $6^{\circ}$ da resolução 001 , de 23 de janeiro de 1986, do Conselho Nacional do Meio Ambiente (CONAMA), como condições básicas de um estudo de impacto ambiental: diagnóstico ambiental; análise dos impactos ambientais; definição das medidas mitigadoras dos impactos negativos; e elaboração do programa de acompanhamento e monitoramento, indicando os fatores e parâmetros a serem considerados (CONAMA 1986a).

Essa resolução do CONAMA prevê a obrigatoriedade da elaboração de Estudo e Relatório de Impacto Ambiental (EIA/RIMA) para o licenciamento de atividades modificadoras do meio ambiente. Por isso, um adequado diagnóstico ambiental do meio físico torna-se imprescindível para a elaboração de um estudo detalhado das condições ambientais da área onde se implantará um dado empreendimento. relatório de controle ambiental (RCA) referente ao licenciamento prévio para perfuração de poços de petróleo do Rio Grande do Norte, porém é observada a ausência de análises e/ou dados geoquímicos como uma das técnicas essenciais na caracterização do diagnóstico do meio físico.

Os estudos geoquímicos são muito utilizados na avaliação de parâmetros de background regional para diagnóstico físico-ambiental, principalmente na quantificação geoquímica dos elementos presentes nos solos, na água e no ar das áreas de influência das atividades de exploração de petróleo, cujos resultados são aplicados no monitoramento e na comparação da situação geoquímica com a legislação ambiental pertinente.

Nosso principal objetivo foi verificar a ausência de análises e dados geoquímicos como parte integrante do diagnóstico ambiental do meio físico, dando ênfase à importância deles para o processo de licenciamento ambiental, além de disponibilizar um roteiro dos estudos geoquímicos e respectivos produtos dentro do diagnóstico ambiental do meio físico, a partir dos levantamentos bibliográficos da geoquímica ambiental e dos estudos de impacto e levantamentos documentais de três relatórios de controles ambientais (RCA), especificamente do item que trata do diagnóstico do meio físico.

$\mathrm{Na}$ elaboração deste trabalho, focamos o diagnóstico ambiental do meio físico, definido no Art. $6^{\circ}$ da resolução CONAMA 001/1986 como:

I - Diagnóstico ambiental da área de influência do projeto, completa descrição e análise dos recursos ambientais e suas interações, tal como existem, de modo a caracterizar a situação ambiental da área, antes da implantação do projeto, considerando: a) o meio físico - o subsolo, as águas, o ar e o clima, destacando os recursos minerais, a topografia, os tipos e aptidões do solo, os corpos d'água, o regime hidrológico, as correntes marinhas, as correntes atmosféricas (CONAMA 1986a).

De acordo com essa resolução, os órgãos estaduais elaboram sua legislação pertinente ao meio ambiente. Para o IDEMA, a lei complementar $n^{\circ} 272$, de 3 de março de 2004:

Regulamenta os artigos 150 e 154 da Constituição Estadual, revoga as Leis Complementares Estaduais n. ${ }^{\circ} 140$, de 26 de janeiro de 1996 , e n. ${ }^{\circ} 148$, de 26 de dezembro de 1996, dispõe sobre a Política e o Sistema Estadual do Meio Ambiente, as infrações e sanções administrativas ambientais, as unidades estaduais de conservação da natureza, institui medidas compensatórias ambientais, e dá outras providências (Rio Grande do Norte 2004).

Para as atividades aqui analisadas, a lei complementar $\mathrm{n}^{\circ}$ 272, de 3 de março de 2004, em seu Art. 47 afirma que: 
Serão exigidas, especificamente, no processo de licenciamento para a perfuração de poços para a identificação ou exploração de jazidas de combustíveis líquidos e gás natural, as seguintes licenças:

I -Licença Prévia para Perfuração - LPPer, concedida para a atividade de perfuração de cada poço, mediante a precedente apresentação, pelo empreendedor, do Relatório de Controle Ambiental (RCA) das atividades e a delimitação da área pretendida (Rio Grande do Norte 2004).

De acordo com o Termo de Referência do IDEMA para a elaboração do relatório de controle ambiental (RCA) referente ao licenciamento prévio para perfuração de poços de petróleo, são considerados para o diagnóstico ambiental do meio físico os seguintes estudos:

Geologia: descrição e mapeamento, na escala 1:25.000, da geologia regional e local, identificando a jazida em mapa geológico;

Geomorfologia local e regional;

Pedologia e aptidão agrícola: descrição de uso dos solos; Recursos Hídricos: caracterização do curso hídrico direta ou potencialmente afetado pelo empreendimento. (Rio Grande do Norte, 2013, p. 2).

Observa-se que não contam nesta lista os estudos geoquímicos, porém eles estão intrínsecos, visto que, dentro do item geologia, a descrição e o mapeamento podem ser realizados com maior precisão quando analisamos, além das características macroscópicas das litologias, as composições químicas do substrato aplicando a técnica de prospecção litogeoquímica e/ou pedogeoquímica.

A geomorfologia de uma área é definida a partir da origem e evolução dos processos geológicos e intempéricos. Eles podem ser estudados a partir do comportamento geoquímico dos elementos que compõem a paisagem geoquímica local e regional, definindo os valores de background que serão utilizados como parâmetro entre o antes e o depois da instalação do empreendimento.

Para o reconhecimento da pedologia e aptidão agrícola, são realizados estudos pedogeoquímicos, e, para os estudos dos recursos hídricos, usa-se hidrogeoquímica. Os resultados serão aplicados na comparação com valores normativos da legislação pertinente.

Desta forma, fica clara a relevância dos estudos geoquímicos nos RCA apresentados ao IDEMA.

No Quadro 1, são apresentados os principais impactos potenciais sobre o meio ambiente da produção de petróleo onshore (Mariano \& Rovere 2006). Observa-se que, dentre os componentes afetados pela atividade petrolífera onshore, o meio físico é o que mais se destaca, desde a seleção do local do empreendimento até o descomissionamento.

No que concerne à delimitação deste trabalho aos relatórios de controles ambientais (RCA), isso ocorreu pois o RCA é o principal estudo ambiental para obtenção de licença prévia para perfuração de poços de petróleo (Sánchez 2008), mais especificamente pelo seu papel no diagnóstico ambiental do meio físico nas etapas de perfuração exploratória e de avaliação.

\begin{tabular}{lccc}
\hline Fonte & Impacto potencial & Ambiente afetado & Comentários \\
\hline Seleção do local & Interações & $\mathrm{H} / \mathrm{B} / \mathrm{Aq}$ & $\begin{array}{l}\text { Sensibilidade considerável em relação à biota, ao uso de recursos, à } \\
\text { importância cultural e à sazonalidade. Impactos secundários relacio- } \\
\text { nados à oferta de equipamentos e a requisitos de suprimentos e im- } \\
\text { pactos potenciais sobre a infraestrutura local. }\end{array}$ \\
\hline
\end{tabular}

Descarga para recursos hídricos - lamas, cascalhos, esgotos, resíduos domésticos, derramamentos e vazamentos. Emissões dos equipamentos das plantas, ruídos e luz, disposição de resíduos sólidos onshore e impactos sobre as infraestruturas locais. Perturbação de organismos terrestres e de aves. Mudanças na qualidade da água, do ar e dos sedimentos. Perda de acesso e perturbação dos outros usuários dos recursos. Emissões e descargas de operações de testes nos poços, descarte de água de produção, combustão e queima em tochas, ruídos e iluminação. De curto prazo e transientes. Efeitos de tráfego de veículos e helicópteros na vida humana e selvagem.

Controles apropriados durante as operações e cuidados no descomissionamento podem, efetivamente, remover riscos de impactos de longo prazo. Controle não apropriado resulta na contaminação de água e sedimentos, danos aos habitats dos organismos e à biodiversidade. Impactos devido à disposição de resíduos sólidos, à infraestrutura e ao conflito no uso dos recursos.

H: humano; B: biosfera; Aq: aquático; At: atmosférico; T: terrestre (Mariano \& Rovere 2006 modificado pelo autor).

Quadro 1

Principais impactos ambientais das atividades petrolíferas onshore, na perfuração exploratória e de avaliação. 


\section{GEOQUÍMICA AMBIENTAL}

A geoquímica ambiental tem como principal campo de estudo a antroposfera. É uma ciência que possui relações interdisciplinares com as ciências naturais e sociais.

O papel das Geociências nos Estudos Ambientais está intimamente ligado à abordagem do meio físico, principalmente relacionado com o entendimento das alterações causadas por processos tecnológicos (obras civis e aproveitamento dos recursos naturais) na dinâmica natural dos processos do meio físico que atuam em determinado ambiente, no qual será desenvolvido o empreendimento (Fornasari Filho \& Bitar 1995).

O diagnóstico ambiental é resultante dos estudos geocientíficos, que necessariamente caracterizam o sistema ambiental potencialmente afetado por empreendimento e/ou atividade poluidora destacando a importância dos estudos geoquímicos (Figura 1).

A Geoquímica é a ciência que estuda a distribuição dos elementos e de seus isótopos nos vários setores orgânicos e inorgânicos da Terra, em superfície e em profundidade (Licht et al. 2007).

Os estudos do meio ambiente vêm sendo contemplados com os conhecimentos dos princípios e das metodologias da geoquímica na identificação e avaliação das ações humanas. Como exemplo, é possível citar as campanhas de amostragem de água e solo que são indicadas, numa certa região, para análises comparativas entre os teores naturais extremamente elevados de elementos químicos dissociados das atividades antrópicas.

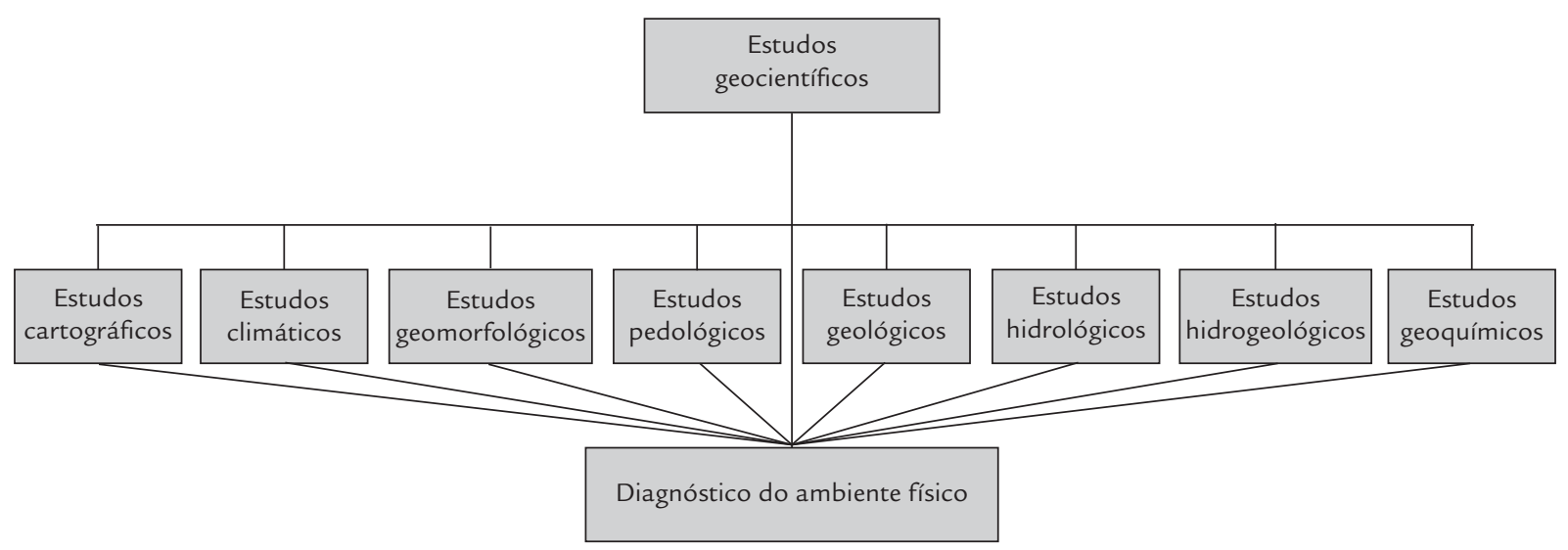

Figura 1

Os estudos ambientais das geociências no diagnóstico do meio ambiente físico

(Rohde 2008 modificado pelo autor.

\subsection{Abrangência da geoquímica ambiental}

A geoquímica ambiental tem sua abrangência evidenciada pelos seguintes objetivos:

- determinar a existência, a concentração, a especiação, a mobilidade potencial e a efetiva migração dos elementos e substâncias de origem antrópica e antropogênica dentro dos diversos compartimentos terrestres;

- em nível dinâmico, o entendimento sistêmico dos processos e ciclos modificados pela ação humana, incluindo a temática da mudança global;

- a avaliação do impacto humano na geoquímica da superfície terrestre, evitando o empobrecimento biológico e socioeconômico associado;

- o aporte de critérios para abordagens legais, reguladoras e de planejamento ambiental;
- a criação de uma perspectiva geoquímica de exploração e uso mais eficiente dos recursos, a implementação de alternativas energéticas e a reciclagem de materiais;

- o aconselhamento, como seu objetivo final, para uma nova ética de bem-estar e racionalismo no uso dos recursos naturais (Rohde 2008).

Podemos ressaltar que a geoquímica ambiental tem como real objetivo estabelecer as principais mudanças ambientais químicas que ocorrem pela intervenção antrópica. Nesse sentido, engrandecemos a relevância que os estudos geoquímicos têm na caracterização do diagnóstico do meio físico, que interfere diretamente na identificação e análise dos impactos ambientais, no programa de monitoramento 
dos impactos ambientais e na tomada de decisão das medidas mitigadoras realmente eficientes.

Estão incluídas na geoquímica ambiental as seguintes técnicas: hidrogeoquímica (estudos das águas superficiais, subterrâneas e os sedimentos de corrente); pedogeoquímica (estudos dos solos e sua gênese); litogeoquímica (estudos das rochas); biogeoquímica (estudos a partir de materiais orgânicos) e a geoquímica a partir de gases.

Nos estudos do meio físico, Rohde (2008) destaca a aplicação de várias outras técnicas como roteiro dos estudos geoquímicos a serem aplicados no diagnóstico do ambiente físico:

- levantamento das emissões contaminantes ${ }^{1}$;

- determinação da origem geológica dos contaminantes;

- análise dos elementos menores e elementos-traço em rejeitos e descargas industriais;

- ensaio de lixiviação e solubilização;

- $\quad$ análise das águas subterrâneas;

- comparações de parâmetros para as águas superficiais;

- pesquisa dos sedimentos de corrente;

- mobilidade dos elementos;

- transporte dos contaminantes;

- caminho vertical e horizontal dos contaminantes (dispersão ambiental);

- microbiogeoquímica:

série NBR ISO 14000 (ABNT/NBR 1996).

\section{RESULTADOS E DISCUSSÕES}

Após a análise dos três RCAs e da legislação pertinente ao tema deste estudo, verificamos que são muitas as problemáticas referentes às ferramentas utilizadas no diagnóstico ambiental.

São vários os trabalhos que discorrem sobre as deficiências dos RCA, tais como Martins e Silveira (2009) analisando o programa de monitoramento ambiental na fase de perfuração; Silva (2011) examinando as deficiências de medidas mitigadoras de um RCA; Sánchez (2008) avaliando os problemas da qualidade dos estudos ambientais e a pobreza na definição do escopo dos estudos ambientais que não levam em consideração os aspectos fundamentais de referência. Para esses relatórios, foi constatado que:

- a consideração de alternativas aos impactos foi negligenciada;

- as medidas mitigadoras propostas muitas vezes eram genéricas e não correspondiam às características do ambiente afetado;

- os planos de monitoramento eram superficiais e não apontavam indicadores;

- há uma carência de procedimentos técnicos adequados para identificar e prever impactos e;

- os procedimentos de valoração e interpretação do significado e importância dos impactos não permite uma avaliação conclusiva (Sánchez 2008).

\footnotetext{
'Elemento ou substância que ocorra no ambiente com concentrações acima das consideradas como sendo os níveis de background (Rohde 2008).
}

- $\quad$ paisagens geoquímicas - Classificação.

- abundância dos elementos e substâncias;

- migração dos elementos - classificação (resistatos; hidróstatos; carbonatos; evaporatos; reduzatos);

- migração dos elementos-holismo;

- fluxos geoquímicos;

- gradientes geoquímicos;

- barreiras geoquímicas.

Como resultado deste roteiro proposto por Rohde (2008), há os seguintes produtos:

- mapas de vulnerabilidade das águas subterrâneas à poluição;

- mapas de dispersão geoquímica;

- determinação dos impactos biogeoquímicos;

- roteiro dos elementos contaminantes;

- mapas das paisagens geoquímicas;

- interpretação teórica e contextualização das situações existentes;

- comparação da situação geoquímica com a resolução CONAMA n ${ }^{\circ}$ 20, de 18 de junho de 1986;

- comparação da situação geoquímica com as normas 10004, 10005 e 10006 (ABNT/NBR 2004a,b,c);

- comparação da situação geoquímica com as normas da

A partir das nossas análises técnicas de três relatórios de controle ambiental (RCA), identificados e cedidos pela coordenação do curso de especialização em licenciamento ambiental de petróleo e gás onshore, fizemos as seguintes observações: $1^{\circ}$ RCA - Perfuração de dois Poços Exploratórios 1-HZ-2-RN e 1-IPU-1-RN Município de Gov. Dix-Sept Rosado (RN) da PROGEL - Projetos Geológicos Ltda. em 2008; $2^{\circ}$ RCA - Perfuração de Dois Poços Exploratórios 1-RAG-4-RN e 1-RAG-5-RN Município de Gov. Dix-Sept Rosado (RN) da PROGEL - Projetos Geológicos Ltda. em 2008: $3^{\circ}$ RCA - Perfuração de Quatro Poços de Petróleo ARG 722-9412-753, ARG 733-9412-753, ARG 745-9412753 e ARG 748-9412-753 no Campo de Alto do Rodrigues Município de Pendências (RN) da ECOPLAM em 2008.

Os três relatórios trazem como caracterização do meio físico o levantamento bibliográfico da geologia regional, arcabouço estrutural, evolução tecno-sedimentar, estratigrafia, geologia local com uma descrição superficial dos principais litotipos encontrados. Não verificamos a relevância da obrigatoriedade da realização desse levantamento.

A geologia deveria ser analisada indicando sua importância para a instalação do empreendimento. A partir da sua caracterização, é possível indicar os locais com menor potencial poluidor: de forma bem genérica, se estivermos trabalhando sobre os terrenos carbonáticos, a infiltração de contaminantes é bem menor do que nos arenitos. A dimensão de uma possível pluma de contaminação pode ser definida com maior precisão, pois conhecemos as características físicas dos dois tipos de terreno e sua capacidade de dispersão. 
A caracterização da geomorfologia não apresenta definições genéticas geoquímicas, mesmo sabendo-se que a geomorfologia está diretamente ligada à sua origem geológica e às ações intempéricas. A forma como os elementos são mobilizados e/ou concentrados em determinados ambientes traz informações pertinentes à delimitação das áreas de influência.

$\mathrm{Na}$ definição da aptidão do solo, somente foram definidas as características macroscópicas e tendo como referência a bibliografia existente. O solo é formado por um conjunto de horizontes com características físicas, químicas e biológicas bem definidas, porém distintas, com capacidades de distribuição e mobilização dos elementos.

O levantamento pedogeoquímico permite traçar um perfil qualitativo com as características naturais atuais do solo e posteriormente compará-las com suas características após a instalação e/ou encerramento do empreendimento, aplicando a legislação pertinente.

Os recursos hídricos superficiais e subterrâneos não são analisados quimicamente, permanecendo desconhecidas suas características físico-químicas e biológicas. Nos levantamentos hidrogeoquímicos, ocorre o mesmo processo de dispersão que nos do solo, sendo ainda mais rápido e abrangente. Sabendo-se que a legislação pertinente ao uso das águas, lei no 9.433, de 8 de janeiro de 1997 (Brasil 1997), é bem mais restritiva do que os demais itens do diagnóstico ambiental, ganha ainda mais relevância uma caracterização física, química e biológica mais especifica das águas superficiais e subterrâneas.

Os responsáveis pelos relatórios abordam o diagnóstico do meio físico a partir das características dos parâmetros físicos, como caracterização do clima e condições meteorológicas, aspectos geológicos, geomorfológicos, pedológicos e recursos hídricos. Mas a metodologia aplicada é a mesma evidenciada nos três RCAs.

Não foi observada a realização de análises químicas que possam ser utilizadas durante a aplicação do plano de monitoramento ambiental e elaboração das medidas mitigadoras. Em todos os textos, não é apresentada nenhuma argumentação que justifique ou sugira a importância dessas informações para o diagnóstico ambiental do meio físico.

No item identificação e análise dos impactos ambientais, são aplicadas tabelas meramente comparativas, sem a presença de análises químicas da qualidade da água, do solo, ou do ar. A ausência de parâmetros normatizados não possibilita a consciência dos possíveis impactos do empreendimento.

\section{CONCLUSÃO E SUGESTÕES}

A partir da análise técnica dos três RCAs para obtenção de licença prévia para perfuração de poços de petróleo onshore no Rio Grande do Norte, observamos que a formulação do diagnóstico do meio físico é tratada apenas como um levantamento bibliográfico, sem a presença dos levantamentos e das análises geoquímicas que possam caracterizar de forma eficiente o meio físico.

$\mathrm{Na}$ realidade, deveriam ser realizadas uma série de estudos geoquímicos, entre os quais:
Apesar da riqueza de imagens e fotografias, é certo que fica comprometida a qualidade do diagnóstico ambiental pela total ausência de informações qualitativas e quantitativas específicas que realmente caracterizem o meio físico, tais como os levantamentos pedogeoquímicos, hidrogeoquímicos, entre outros.

Evidenciamos nos três RCAs o quanto é superficial o diagnóstico ambiental do meio físico. Eles trazem um mero levantamento bibliográfico, no qual não são destacadas as características e aptidões químicas dos terrenos geológicos, da qualidade do ar, da água e dos solos.

Esse fato possivelmente prejudica o monitoramento e todas as etapas seguintes do processo dos estudos de impactos ambientais.

Rohde (2008) enfatiza a importância dos estudos geoquímicos para a avaliação dos impactos ambientais, principalmente na interpretação teórica e contextualização das situações existentes, na comparação da situação geoquímica com a resolução CONAMA 20/1986 (CONAMA 1986b), as normas 10004, 10005 e 10006 (ABNT/NBR 2004a, 2004b e 2004c) e as normas da série NBR ISO 14000 (ABNT/ NBR 1996).

Os três RCA analisados apresentam uma discrepância com o que a legislação ambiental sugere como o mínimo exigido para a caracterização dos impactos ambientais:

Artigo $1^{\circ}$ - Para efeito desta Resolução, considera-se impacto ambiental qualquer alteração das propriedades físicas, químicas e biológicas do meio ambiente, causada por qualquer forma de matéria ou energia resultante das atividades humanas que, direta ou indiretamente, afetam:

I - a saúde, a segurança e o bem-estar da população;

II - as atividades sociais e econômicas;

III - a biota;

IV - as condições estéticas e sanitárias do meio ambiente; $\mathrm{V}$ - a qualidade dos recursos ambientais (CONAMA 1986a).

Como é o órgão estadual (IDEMA) que define o termo de referência para as atividades petrolíferas, observou-se que a definição do diagnóstico ambiental do meio físico é bastante genérica. Isso contribui para a falta de detalhamento dos estudos necessários para a caracterização dos potenciais impactos ambientais das atividades petrolíferas do Rio Grande do Norte.
- definição da paisagem geoquímica da região ou área de influência direta e indireta;

- levantamento das emissões contaminantes antes e após a implantação da atividade, facilitando a elaboração e implantação do Plano de Monitoramento Ambiental (PMA) e a definição das medidas mitigadoras;

- análise dos elementos menores e elementos-traço em rejeitos e descargas industriais; 
- $\quad$ ensaio de lixiviação e solubilização dos resíduos industriais;

- análise das águas subterrâneas a partir das amostras hidrogeoquímicas;

- comparações de parâmetros para as águas superficiais previstas na legislação ambiental;

- avaliação da mobilidade dos elementos para comparação dos efeitos da atividade implantada sobre o meio físico;

- pesquisa sobre como ocorre o transporte dos contaminantes no meio físico e quais as suas consequências;

- definição dos principais caminhos vertical e horizontal dos contaminantes (dispersão ambiental), que estão intimamente ligados ao tipo de substrato geológico;

- levantamento microbiogeoquímico da área para monitoramento da qualidade do solo, das águas e do ar.

Como sugestão, a partir destes estudos geoquímicos seria realizada a comparação com as situações geoquímicas normatizadas pertinentes a cada item do diagnóstico ambiental do meio físico. Ou seja, ocorreria a avaliação real da qualidade das águas, dos solos, do ar e da paisagem

\section{AGRADECIMENTOS}

Agradecemos ao Programa de Mobilização da Indústria Nacional de Petróleo e Gás Natural (PROMIMP) e ao Instituto Federal de Educação, Ciência e Tecnologia do geoquímica da área de influência direta e indireta do empreendimento antes e após sua instalação. As informações vão subsidiar a elaboração do plano de monitoramento ambiental e definição das medidas mitigadoras.

O Termo de Referência para os relatórios de controle ambientais deveria trazer mais detalhes para a elaboração do RCA, definindo melhor como realmente pode ser caracterizada a análise dos impactos ambientais.

As distorções na realização do diagnóstico ambiental têm influência direta em todas as etapas dos estudos ambientais, principalmente no plano de monitoramento ambiental e na tomada de decisão quanto às medidas mitigadoras. O diagnóstico ambiental tem grande relevância, além de ser o objeto utilizado na tomada de todas as decisões ambientais e econômicas do empreendimento.

Todas essas sugestões partem do questionamento de como podemos analisar os impactos ambientais se não temos conhecimento suficiente do seu estado natural na área do empreendimento, anterior à implantação de qualquer atividade potencialmente poluidora.

Rio Grande do Norte (IFRN) por propiciar qualificação específica para nossa área de atuação dentro da diretoria de recursos naturais.

\section{REFERÊNCIAS}

ABNT - Associação Brasileira de Normas Técnicas. 1996. NBR ISO 14000 -

Família de normas ISO 14000. ABNT, Rio de Janeiro.

2004a. NBR 10004 - Resíduos sólidos - classificação. ABNT, Rio de Janeiro, 71 p.

ABNT - Associacao Brasileira de Normas Tecnicas. 2004b. NBR 10005 -

Procedimento para obtenção de extrato lixiviado de resíduos sólidos. ABNT, Rio de Janeiro, 3 p.

ABNT - Associacao Brasileira de Normas Tecnicas. 2004c. NBR 10006 Procedimento para obtenção de extrato solubilizado de resíduos sólidos, $2^{a}$ ed. ABNT, Rio de Janeiro, 3 p.

Brasil. 1997. Lei n ${ }^{\circ}$ 9.433, de 8 de janeiro de 1997. Institui a Política Nacional de Recursos Hídricos, cria o Sistema Nacional de Gerenciamento de Recursos Hídricos e dá outras providências. Código Civil, Brasília, 1997. Disponível em: http://www.planalto.gov.br/ccivil_03/leis/L9433.htm. Acessado em 07 fev 2013.

CONAMA - Conselho Nacional do Meio Ambiente. 1986a. Resolução CONAMA $\mathrm{n}^{\circ}$ 1, de 23 de janeiro de 1986. Disponível em: http://www.mma.conama.gov. br/port/conama. Acessado em 25 jan 2013.

CONAMA - Conselho Nacional do Meio Ambiente. 1986b. Resolução CONAMA n ${ }^{\circ} 20$ de 18 de junho de 1986. Disponível em: http://www.mma. conama.gov.br/port/conama. Acessado em 5 fev 2013.

ECOPLAM - Empresa de Consultoria e Planejamento Ambiental Ltda. 2008. Perfuração de Quatro Poços de Petróleo ARG 722-9412-753, ARG 7339412-753, ARG 745-9412-753 e ARG 748-9412-753 no Campo de Alto do Rodrigues Município de Pendências (RN). Natal, Ecoplam. 1 CD-ROM.

Fornasari Filho N. \& Bitar O.Y. 1995. O meio físico em estudos de impacto ambiental-EIAs. In: O.Y. Bitar (coord.). Curso de geologia aplicada ao meio ambiente. Associação Brasileira de Geologia de Engenharia (ABGE) e Instituto de Pesquisas Tecnológicas (IPT), São Paulo, capítulo 4.1, p.151-163. 
Licht O.A.B., Mello C.S.B., Silva C. R. 2007. Prospecção geoquímica: Depósitos minerais metálicos, não-metálicos, óleo e gás. SBGq/CPRM, Rio de Janeiro, 788 p.

Mariano J.B. \&, Rovere E.L.L. 2006. Impactos ambientais da exploração e produção de petróleo em áreas offshore. In: IBP, Rio Oil \& Gas Expo and Conference, Anais, v. II. p. 577-588.

Martins R.A. \& Silveira I.M. 2009. Implementação do programa de monitoramento ambiental na fase de perfuração: estudo de caso no município de Governador Dix-Sept Rosado (RN). In: E.A.C. Pegado, V.P. Silva (orgs.), Licenciamento ambiental onshore: limites e otimização. IFRN, Natal, p. 115-129.

Matos A.T. 2010. Poluição ambiental: Impactos no meio físico. UFV, Viçosa, 260 p.

Pfaltzgraff P.A.S. \& Torres F.S.M. 2010. Geodiversidade do Rio Grande do Norte. CPRM, Recife, 227 p.

PROGEL - Projetos Geológicos Ltda. 2008a. Perfuração de Dois Poços Exploratórios 1-HZ-2-RN e 1-IPU-1-RN Município de Gov. Dix-Sept Rosado (RN). Mossoró, PROGEL. 1 CD-ROM.

PROGEL - Projetos Geologicos Ltda. 2008b. Perfuração de Dois Poços Exploratórios 1-RAG-4-RN e 1-RAG-5-RN Município de Gov. Dix-Sept Rosado-RN. Mossoró, Progel. 1 CD-ROM.

Rio Grande do Norte. 2014. Lei complementar n 272, de 3 de março de 2004. Dispõe sobre a Política e o Sistema Estadual do Meio Ambiente, as infrações e sanções administrativas ambientais e dá outras providências. Natal, 2004. Disponível em: http://www.idema.rn.gov.br. Acessado em 20 jan 2013.

Rio Grande do Norte. 2013. Instituto de Desenvolvimento Sustentável e Meio Ambiente. Termo de referência: elaboração de RCA referente ao licenciamento prévio para a perfuração de poços petrolíferos. Disponível em: http://www. idema.rn.gov.br. Acessado em 20 jan 2013.

Rohde G.M. 2008. Geoquímica ambiental e estudos de impactos. 3. ed. Signus, São Paulo, 184 p.

Sánchez, L.E. 2008. Avaliação de impactos ambientais: conceitos e métodos. Oficina de textos, São Paulo, 496 p.

Silva R.G. 2011. As deficiências de medidas mitigadoras de um RCA que compromete o princípio do desenvolvimento sustentável. Revista Holos, 27(1):72-91. 